\title{
NOÇÃO TEMPORAL DE CRIANÇAS COM DESENVOLVIMENTO TÍPICO E TRANSTORNO DO DESENVOLVIMENTO DA COORDENAÇÃO
}

\author{
Rafaela Zortéa Fernandes Costa ${ }^{1} \mathbb{D}$; Inara Marques ${ }^{1} \mathbb{D}$; Dalberto Luiz de Santo ${ }^{1} \mathbb{D}$; Josiane Medina-Papst ${ }^{1} \mathbb{D}$ \\ RESUMO
}

O objetivo do estudo foi comparar o desenvolvimento da noção de tempo entre crianças com Desenvolvimento Típico (DT) e com Transtorno do Desenvolvimento da Coordenação (TDC). Participaram 34 crianças, divididas nos grupos: DT 6-7 anos ( $n=9)$, DT 8-9 anos ( $n=8)$, TDC 6-7 anos $(n=7)$ e TDC 8-9 $(n=10)$. As crianças com TDC foram indicadas pelos professores por meio do checklist do MABC-2 e as crianças com DT por apresentarem bom desempenho. Posteriormente, todas foram avaliadas com a bateria do MABC-2. Para a análise da noção temporal, utilizou-se uma adaptação de uma prova proposta por Piaget, que visa compreender a sucessão dos acontecimentos percebidos, realizada em contexto experimental. Sendo assim, utilizaram-se dois brinquedos de madeira que realizavam movimentos distintos em relação ao tempo e ao ponto de chegada. O conceito dessa tarefa foi adaptado para duas tarefas de corrida em contexto aplicado. As crianças foram questionadas separadamente sobre questões temporais das tarefas e suas respostas gravadas e transcritas. Empregou-se o teste Mann-Whitney, observando-se que as crianças com TDC expressaram níveis operatórios de pensamento, não demonstrando diferença significativa entre grupos.

Palavras-chave: Desenvolvimento infantil; Habilidades motoras; Transtornos motores.

\section{The concept of time in children with typical motor development and developmental coordination disorder}

\begin{abstract}
The objective of the study was to compare the development of the notion of time between children with Typical Development (TD) and with Developmental Coordination Disorder (DCD). In this research 34 children participated, divided into groups: DT 6-7 years $(n=9)$, DT 8-9 years $(n=8)$, TDC 6-7 years $(n=7)$ and TDC $8-9(n=10)$. Children with BDD were indicated by teachers through the MABC-2 checklist and children with TD for presenting a good performance. Subsequently, all of them were evaluated using the MABC- 2 battery. For the analysis of the temporal notion, an adaptation of a test proposed by Piaget was used, which aims to understand the succession of perceived events, carried out in an experimental context. Therefore, two wooden toys were used that performed different movements in relation to time and arrival point. The concept of this task was adapted for two running tasks in an applied context. The children were asked separately about the task's temporal issues and their recorded and transcribed responses. The Mann-Whitney test was used, noting that children with DCD expressed operative levels of thought, showing no significant difference between groups.
\end{abstract}

Keywords: Cognitive development; Motor skills; Motor Disorders.

\section{Noción temporal de niños con desarrollo típico y trastorno del desarrollo de la coordinación \\ RESUMEN}

El objetivo del estudio fue comparar el desarrollo de la noción de tiempo entre niños con Desarrollo Típico (DT) y con Trastorno do Desarrollo de la Coordinación (TDC). Participaron 34 niños, divididos en los grupos: DT 6-7 años ( $n=9$ ), DT 8-9 años $(n=8)$, TDC 6-7 años $(n=7)$ y TDC 8-9 $(n=10)$. Los niños con TDC fueron indicadas por los profesores por intermedio del checklist del $M A B C-2$ y los niños con DT por presentar buen rendimiento. Posteriormente, todos fueron evaluadas con la batería del $M A B C$-2. Para el análisis de la noción temporal, se utilizó una adaptación de una prueba propuesta por Piaget, que tiene por objetivo comprender la sucesión de los acontecimientos percibidos, realizada en contexto experimental. Siendo así, se utilizaron dos juguetes de madero que realizaban movimientos distintos en relación al tiempo y al punto de llegada. El concepto de esa tarea fue adaptado para dos tareas de corrida en contexto aplicado. Se indagó, a los niños, separadamente sobre cuestiones temporales de las tareas y sus respuestas gravadas y transcritas. Se empleó el test Mann-Whitney, observándose que los niños con TDC expresaron niveles operatorios de pensamiento, no demostrando diferencia significativa entre grupos.

Palabras clave: Desarrollo infantil; destreza motora; Trastorno motores.

1 Universidade Estadual de Londrina - Londrina - PR - Brasil; rafaela_zortea@hotmail.com; inaramarques@hotmail.com; dalbertodesanto@gmail.com; josi_medina@hotmail.com 


\section{INTRODUÇÃO}

É reconhecido pela teoria de desenvolvimento cognitivo que, por volta dos sete anos de idade, a criança inicie o Estágio das Operações Intelectuais Concretas (Piaget, 1958), sendo esse estágio marcado por uma passagem decisiva da ação para a operação (Piaget, 1978). Assim como nos outros estágios do desenvolvimento cognitivo, no Estágio das Operações Concretas as noções passam por transformações, construindo estruturas completamente novas. Contudo, os obstáculos vencidos para a construção das operações, trazem, concomitantemente, o desenvolvimento altamente complexo de novas noções, tais como o tempo, a velocidade, a causalidade, entre outros, antes compreendidos como simples esquemas de ações e intuições. Estas noções fornecem informações para que o indivíduo estabeleça relações entre os objetos que se deslocam no espaço (Rosa Neto, 2002), relações que, a todo o momento, são infinitamente necessárias tanto em tarefas cotidianas, quanto nas esportivas ou recreativas como, por exemplo, para se atravessar uma rua, ou para se calcular a distância e a velocidade necessária para interceptar um objeto ou uma bola lançada em nossa direção.

Um dos primeiros pesquisadores a se preocupar com essas questões foi Piaget, que realizou, em contexto experimental, diversos estudos do desenvolvimento dessas noções ao longo da infância. Geralmente, nesses estudos, cada criança sentava-se à mesa de experimento e observava os materiais e situações apresentados a ela e fornecia respostas aos questionamentos propostos pelo pesquisador. Continuando com essa problemática em mente, mas a partir da perspectiva do profissional de Educação Física no contexto educacional, que almeja o desenvolvimento tanto de aspectos motores quanto cognitivos dos alunos, questionou-se inicialmente como ocorre essa representação da noção de tempo, quando a criança sai da posição de mera observadora do movimento de objetos e passa a ser a executora do movimento que observou.

Essa ligação necessária do desenvolvimento cognitivo, a partir das experiências vividas, é um desafio para o educador, dado que, diferente do pesquisador, ele visa o aprendizado contínuo de seus alunos, a partir de situações nas quais os alunos atuem sobre os elementos e informações do ambiente, mas também pode ser um obstáculo quando se esbarra em características individuais, como as próprias limitações motoras das crianças. Assim, nessa perspectiva de análise conjunta do desenvolvimento motor e cognitivo, nota-se que a idade aproximada de sete anos é um marco desenvolvimental nas mudanças observadas no ciclo da infância. É em torno dessa idade que se espera que a criança inicie a combinação das habilidades motoras fundamentais (Manoel, 1994), marcada pela necessidade de integração entre duas habilidades, que, por sua vez, só ocorre a partir de uma modificação e reorganização das estruturas espaciais e temporais dessas para que seja possível ao executante antecipar a próxima sequência na ação (Gimenez, Manoel, Oliveira, Dantas, \& Marques, 2012; Tani, Basso, \& Corrêa, 2012). Isso significa dizer que, por exemplo, habilidades como chutar, correr, saltar, girar, entre outras, quando combinadas entre si, serão modificadas espacial e temporalmente, de modo que possam ser aplicadas no esporte, no lazer e, principalmente, nos eventos da vida diária. Além disso, para que se alcance essa maior complexidade na realização das habilidades, o indivíduo necessita de padrões de movimentos maduros em uma grande variedade de habilidades motoras. Entretanto, hoje, sabe-se que algumas crianças, apesar de, aparentemente, não apresentarem nenhum comprometimento ao longo do desenvolvimento, apresentam dificuldades para realizar tarefas que demandam habilidades motoras finas e amplas - expressas em termos como "descoordenadas", "desajeitadas" etc.

A literatura aponta que, aproximadamente $6 \%$ de crianças em idade escolar, entre 5 e 11 anos de idade, possuem um acentuado comprometimento no processo de desenvolvimento motor (Associação Psiquiátrica Americana [APA], 2014). Essas crianças têm dificuldades em realizar tarefas que demandam coordenação motora fina e ampla, tanto em atividades da vida diária como de recreação. Em 1994, a Associação Psiquiátrica Americana propôs a utilização de um termo que pudesse expressar as dificuldades motoras observadas no desempenho dessas crianças. Com isso, o termo "Transtorno do Desenvolvimento da Coordenação" (TDC), como descrito no Manual Diagnóstico e Estatístico de Transtornos Mentais (DSM-V) (APA, 2014), passou a ser adotado nos estudos das diversas áreas de conhecimento, como na educação e na saúde.

Há algum tempo, diversas áreas de estudo vêm buscando compreender como ocorre o desenvolvimento dessa população específica e, assim, muitos pesquisadores têm contribuído com pesquisas, principalmente para que seja encontrada alguma solução para mitigar os impactos decorrentes do transtorno. Além das dificuldades motoras, sabe-se que crianças com problemas de coordenação motora apresentam dificuldades tanto em habilidades espaciais quanto em tarefas com demanda temporal (Estil, Ingvaldsen, \& Whiting, 2002). A literatura também aponta que o TDC, geralmente, está associado a problemas de saúde mental, como depressão e ansiedade (Omer, Jijon, \& Leonard, 2018), problemas com autoconceito de competência física (Yu et al., 2016), baixa qualidade de vida (Cleaton, Lorgelly, \& Kirby, 2019), baixa interação social (Poulsen, Ziviani, Johnson, \& Cuskelly, 2008) e Transtornos de Hiperatividade (Okuda, 2015).

Porém, atualmente, poucos estudos buscam com- 
preender o comportamento das crianças com TDC no âmbito escolar ou as mudanças no desenvolvimento cognitivo dessa população (Roebers \& Kauer, 2009). Nota-se ainda que, apesar do aumento de estudos durante esse período, poucos professores atuantes na escola conhecem esse tipo de transtorno e, tampouco, estratégias para que possam amenizar as dificuldades dessas crianças. De acordo com Santos et al. (2015), é importante para os profissionais de Educaçãoo Física a identificaçãoo de crianças com TDC, fornecendo informações importantes para o planejamento de estratégias e intervenções motoras, com intuito de minimizar o impacto decorrente desse transtorno. Nesse sentido, por ser a Educação Física uma disciplina escolar que atua sobre a educação motora em todas as suas vertentes, os profissionais desta área devem estar atentos e preparados para identificar e agir adequadamente a partir das possíveis dificuldades motoras de seus alunos.

Sendo assim, pensando em uma educação que almeja auxiliar cada criança a vencer suas dificuldades, há a necessidade de pesquisas que busquem investigar a relação entre o TDC e as mudanças que ocorrem no mecanismo cognitivo dessas crianças, possibilitando o futuro desenvolvimento de melhores ambientes de aprendizagem. Procurando contribuir nesta direção, o objetivo deste trabalho foi de comparar como ocorre o desenvolvimento da noção de tempo em crianças caracterizadas com Desenvolvimento Típico (DT) e com Transtorno do Desenvolvimento da Coordenação (TDC), tanto em situações de observação do movimento de objetos como de execução de tarefas motoras. Como pressuposto do estudo, postulou-se que, dentro da mesma faixa etária e nas duas situações propostas, crianças com DT apresentariam melhor compreensão da noção de tempo quando comparadas as crianças com TDC.

\section{MÉTODO}

Este estudo caracteriza-se por um estudo de campo, observacional e qualitativo (Thomas, Nelson, \& Silverman, 2012).

\section{Participantes}

As crianças participantes desta pesquisa estudavam em duas escolas da Região Metropolitana de Londrina. Para selecioná-las, inicialmente solicitou-se aos professores de Educação Física e regentes de sala que preenchessem o checklist do MABC-2 - instrumento de triagem que visa observar o comportamento de uma criança em diversas situações - para cada criança que eles entendiam ter dificuldades motoras nas atividades da escola. Após a indicação das crianças, elas foram avaliadas pela pesquisadora com a bateria de testes do MABC-2, composta por atividades que avaliam habilidades nas áreas de destreza manual, equilíbrio dinâmico e estático e habilidades com bola (Henderson, Sugden, \& Barnett, 2007). Num segundo momento, solicitou-se aos professores de educação física que indicassem crianças com desenvolvimento típico, caracterizado por apresentarem bom desempenho motor em tarefas referentes à manipulação de objetos, equilíbrio estático e dinâmico e jogos com bola. Posteriormente, essas crianças também foram avaliadas pela pesquisadora nas tarefas do MABC-2 para descartar qualquer dificuldade motora que as impedissem de participar do estudo.

Dado que os indivíduos são avaliados com TDC ou DT segundo os graus de um ou outro comportamento que apresentam, a amostra foi formada por conveniência, adotando o percentil igual ou menor que 9 como ponto de corte para a classificação de TDC e percentil igual ou superior a 63 como ponto de corte para a seleção das crianças com DT.

Após esses procedimentos iniciais, participaram do estudo 34 crianças ( 25 meninos e 9 meninas), entre 6 e 9 anos, divididas em quatro grupos: Desenvolvimento Típico 6-7 anos (DT 6-7 anos; $n=9$ ), Desenvolvimento Típico 8-9 anos (DT 8-9 anos; $n=8$ ), Transtorno do Desenvolvimento da Coordenação 6-7 anos (TDC 6-7 anos; $\mathrm{n}=7$ ) e Transtorno do Desenvolvimento da Coordenação 8-9 anos (TDC 8-9 anos; $n=10$ ), conforme Tabela 1.

Os pais ou responsáveis assinaram um termo de consentimento livre e esclarecido autorizando a participação das crianças no estudo. A pesquisa foi aprovada pelo Comitê de Ética em Pesquisas com Seres Humanos da Universidade Estadual de Londrina (Parecer no 56826).

\section{Tarefas}

As tarefas utilizadas para investigar o desenvolvimento da noção de tempo das crianças foram baseadas em uma prova utilizada por Piaget (2012) para analisar a sucessão dos acontecimentos percebidos. Para isso, neste estudo, as tarefas foram realizadas de duas for-

Tabela 1. Número de crianças participantes da amostra.

\begin{tabular}{|c|c|c|c|c|c|c|}
\hline & \multicolumn{2}{|c|}{ 6-7 anos } & \multicolumn{2}{|c|}{ 8-9 anos } & \multicolumn{2}{|c|}{ Total } \\
\hline & $\mathbf{N}$ & $\%$ & $\mathbf{N}$ & $\%$ & $\mathbf{N}$ & $\%$ \\
\hline DT & 9 & $26,47 \%$ & 8 & $23,53 \%$ & 17 & $50,00 \%$ \\
\hline TDC & 7 & $20,59 \%$ & 10 & $29,41 \%$ & 17 & $50,00 \%$ \\
\hline Total & 16 & $47,06 \%$ & 18 & $52,94 \%$ & 34 & $100 \%$ \\
\hline
\end{tabular}

Fonte: próprio autor. 
mas: em contexto experimental (CE), conforme proposto por Piaget, e em contexto aplicado (CA). Optou-se por realizar a tarefa também em CA, tendo em vista que, desta forma, a tarefa ficaria mais próxima do contexto de uma aula de Educação Física, possibilitando este tipo de análise pelos professores.

As crianças foram avaliadas no próprio ambiente escolar pela pesquisadora, em sala previamente preparada, em dia e horário agendados anteriormente com a coordenação. Para iniciar as tarefas, explicava-se a cada criança que seria realizada uma tarefa na sala (Contexto Experimental-CE) e duas tarefas fora da sala juntamente com outra criança (Contexto Aplicado-CA). Antes do início da tarefa de $\mathrm{CE}$, cada criança foi instruída verbalmente a ficar atenta ao material exposto que seria utilizado no experimento. Após as explicações, se ela apresentasse alguma dúvida era permitido observar o material mais uma vez, caso contrário, a tarefa era iniciada.

No contexto experimental (CE), adaptando-se o estudo de Piaget (2012) sobre a sucessão dos acontecimentos percebidos, foi apresentado à criança um brinquedo de madeira que consistia em dois patinhos amarelos - o patinho I e o II - sendo que o segundo continha um ponto vermelho para diferenciá-lo do primeiro. Cada patinho foi posicionado no início de duas rampas paralelas e retilíneas de $48 \mathrm{~cm}$ de comprimento e inclinação de $23^{\circ}$ em relação à mesa de suporte.

Os patinhos percorriam o seu caminho iniciando a descida ao mesmo tempo, mas por possuírem diferentes comprimentos de passada atingiam velocidades diferentes. Sendo assim, enquanto o patinho I (maior velocidade) percorria os $48 \mathrm{~cm}$ de distância da rampa, o patinho II (menor velocidade) percorria somente 22 $\mathrm{cm}$ de distância. No momento que o patinho I parava ao final do seu percurso $(48 \mathrm{~cm})$, o patinho II continuava percorrendo mais $15 \mathrm{~cm}$ na rampa, parando apenas no ponto em que uma fita dupla face fora colocada, completando $37 \mathrm{~cm}$ de percurso total.

Em seguida, a criança foi questionada com as seguintes perguntas: (1) Os dois patinhos pararam ao mesmo tempo?; (2) Se não, qual parou primeiro?; (3) Qual patinho andou mais tempo?; e (4) Se imaginássemos que o patinho I parou ao meio dia, então o patinho II parou antes, depois ou ao meio dia? Ainda, no decorrer das perguntas as crianças foram questionadas do porquê de suas respostas. O termo "meio dia" foi utilizado para manter os questionamentos sobre a tarefa da mesma maneira utilizada por Piaget (2012).

Para a avaliação em contexto aplicado (CA), os conceitos de tempo, velocidade, e causalidade da primeira tarefa foram adaptados a um contexto dinâmico, sendo elaboradas duas atividades. Nas duas atividades as crianças do mesmo grupo etário participaram em du- plas, sendo uma criança com DT e uma criança com TDC.

A primeira tarefa de CA, portanto, consistiu em apresentar às crianças dois trajetos: um com distância de 5 metros e um com distância de 4 metros, que foram montados no pátio ou quadra da escola. Os trajetos foram compostos por cones enfileirados, distribuídos a um metro de distância um do outro e um arco posicionado a um metro do final do percurso.

As crianças foram instruídas a correr pelos trajetos em ziguezague, o mais rápido possível e, ao final, lançarem uma peteca no arco. Em seguida, as crianças foram questionadas separadamente se: (1) Elas pararam ao mesmo tempo?; (2) Quem parou primeiro?; (3) Quem andou mais tempo?; e (4) Se a criança $X$ (que chegou primeiro) parou ao meio dia, a criança $Y$ (que chegou depois) parou antes, depois ou ao meio dia?

A segunda tarefa de CA consistiu em apresentar dois trajetos, com a distância de 5 metros. Em um dos trajetos foram colocados arcos enfileirados de modo que a criança deveria pisar somente dentro dos arcos, e no outro trajeto estavam dispostos cones de modo que ela percorresse em ziguezague. As duas crianças percorreriam uma vez cada trajeto, portanto, foi distribuída a tarefa em duas etapas.

Elas foram instruídas a percorrer os trajetos o mais rápido possível e, ao chegar ao final, deveriam chutar uma bola ao gol. As crianças foram questionadas separadamente ao final de cada etapa, se: (1) Elas pararam ao mesmo tempo?; (2) Quem parou primeiro?; (3) Quem andou mais tempo?; e (4) Se a criança $X$ (que chegou primeiro) parou ao meio dia, a criança $Y$ (que chegou depois) parou antes, depois ou ao meio dia?

Para cada dupla de crianças, as tarefas e atividades foram conduzidas e filmadas no mesmo dia, para análise posterior.

\section{Procedimentos de análise}

As tarefas foram filmadas utilizando-se uma câmera digital da marca Sony, modelo Handycam DCR-SR42, com frequência de amostragem de $60 \mathrm{~Hz}$. As respostas das crianças sobre os questionamentos foram transcritas e os vídeos analisados de acordo com a teoria Piagetiana sobre o desenvolvimento da noção de tempo, para posterior categorização das respostas dentro das etapas previstas na teoria (etapa 1 , subetapa $2 \mathrm{~A}$, subetapa $2 \mathrm{~B}$ e etapa 3).

Dessa forma, na primeira etapa (E1) encontram-se os sujeitos que apresentaram sucessões temporais e espaciais indiferenciadas, determinadas por respostas contraditórias quanto à duração e a sucessão dos acontecimentos, não comportando assim nenhuma lógica. Todas as relações que a criança faz do tempo ficam indiferenciadas do espaço e também da velocidade. A criança possui dificuldades em, por exemplo, avaliar o 
"antes" e o "depois" de uma forma temporal. A duração, neste nível, seria assimilada somente na velocidade e no espaço percorrido (Piaget, 2012).

A segunda etapa é separada em duas subetapas, de acordo com as mudanças relacionadas ao desenvolvimento cognitivo da criança. A subetapa $2 A$ (S2A) diz respeito ao começo da diferenciação entre a ordem temporal e a ordem espacial e intuições temporais articuladas. As crianças categorizadas nesta etapa apresentam um grande progresso. Contudo, alguns sujeitos apresentam uma melhor compreensão da noção de sucessão, enquanto outros apresentaram uma melhor compreensão da duração. Quando há progresso da noção de sucessão, a criança explica que quem andou "mais tempo" andou "mais rápido" ou "mais longe", contudo ela diferencia sem dificuldade quem chegou "antes" ou "depois". Já, quando há progressos da noção de duração a criança explica sem dificuldades que andar "mais tempo" significa ter percorrido mais lentamente determinado percurso, porém compreende as relações de "antes" e "depois" ainda de forma espacial.

A subetapa 2B (S2B) é denominada como o começo da coordenação operatória entre as intuições articuladas. A grande característica desta etapa é que a criança começa a descobrir a necessidade de fundar as relações de sucessão nas de duração e vice-versa. Portanto, as crianças deste grupo, na medida em que vão sendo questionadas, gradativamente vão corrigindo suas respostas.

A terceira etapa (E3) consiste na sucessão e duração operatória. Diferentemente das etapas anteriores, os sujeitos desta etapa reagem desde o início segundo uma lógica operatória, levando em consideração diferentes fatores como os pontos de partida e de parada do objeto que se desloca, demonstrando uma verdadeira compreensão temporal e espacial - e da qual se infere o domínio da capacidade de descentração do seu pensamento (Piaget, 2012).

Todos os procedimentos de avaliação das crianças, aplicação das tarefas e análise dos dados foram realizados pela própria pesquisadora. Após a categorização das crianças, os resultados foram analisados quantitativamente por meio de porcentagem e qualitativamente em relação ao tipo e a profundidade das explicações fornecidas. Por fim, para a avaliação dos pressupostos do estudo, utilizou-se o teste $U$ de Mann-Whitney para a comparação entre os grupos e entre as etapas do mesmo grupo. Adotou-se o nível de significância de $p<0,05$ e o pacote estatístico SPSS for Windows, versão 13.0.

\section{RESULTADOS}

Dentre as 34 crianças avaliadas neste estudo, 20,59\% foram categorizadas na Primeira Etapa (E1), $38,24 \%$ categorizadas na subetapa $2 A$ (S2A), $23,53 \%$ na subetapa 2B (S2B) e 17,65\% na Terceira Etapa (E3). Em relação aos grupos, observou-se que a maior parte das crianças com TDC (70,59\%) foi categorizada nas etapas E1 e S2A, por outro lado, a maior parte das crianças com DT $(64,70 \%)$ foi categorizada nas S2A e S2B (Tabela 2).

Quanto à análise por idade, os resultados apontaram que nenhuma criança com TDC de 6-7 anos foi categorizada nas etapas S2B e E3, concentrando-se a maior parte na primeira etapa do desenvolvimento cognitivo. Já as crianças com DT dessa idade distribuíram-se pelas três etapas do desenvolvimento, com maior porcentagem classificada na etapa 2.

$\mathrm{Na}$ idade de 8-9 as crianças com TDC classificaram-se da etapa S2A (50,0\%) até a etapa E3 do desenvolvimento (30,0\%). Por outro lado, o desempenho das crianças com DT se distribuiu de modo mais uniforme entre as etapas, com maior frequência na subetapa S2B $(37,5 \%)$ e E3 (25,0\%).

Embora os dados aparentem diferentes distribuições entre os grupos TDC e DT, o teste $U$ de Mann-Whitney não apresentou diferença estatisticamente significante entre eles $(U=118,5 ; p=0,375)$. Dessa forma, realizou-se o teste $U$ de Mann-Whitney entre as etapas do desenvolvimento dentro de cada grupo etário, não encontrando diferença significante nesta análise $(U=14,500 ; p=0,071$ no grupo 6-7 anos e $U=40,00 ; p=1,00$ no grupo $8-9$ anos). Por fim, ao ser feita a comparação entre grupos etários, independente de TDC ou DT, verificou-se diferença estatística, com as crianças mais novas classificando-se nas primeiras etapas do desenvolvimento da noção de tempo, e as mais velhas nas etapas finais $(U=77,0 ; p=0,02)$.

\section{DISCUSSÃO}

Os resultados encontrados permitiram inferir que as crianças, tanto no contexto experimental (CE) quanto no contexto aplicado (CA), adotaram a mesma lógica de pensamento, pois a criança categorizada em uma dada etapa no contexto experimental da tarefa permaneceu nesta mesma etapa quando a tarefa foi realizada em contexto aplicado. Com isso, assumimos que a lógica para a construção da noção de tempo pela criança não se alterou quando da passagem da posição de observador para a função de executor das tarefas.

Em relação à hipótese de estudo estabelecida, na qual as crianças com DT apresentariam melhor compreensão da noção de tempo quando comparadas às crianças com TDC, os resultados não permitiram a sua confirmação. Entretanto, os resultados do presente estudo demonstraram que o maior número de crianças com TDC foi categorizado nas etapas E1 e S2A (70,59\%), enquanto que o maior número de crianças com DT foi categorizado na S2A e S2B $(64,70 \%$ ) (Tabela 2). Conforme Piaget (2012) apontou em seus estudos, a passagem da S2B para a E3 é um período de transição muito mais rápido em relação à passagem da E1 para a S2A. Isso ocorre porque a S2B é uma passagem do pensamento 
Tabela 2. Número de crianças classificadas em cada etapa.

\begin{tabular}{|c|c|c|c|c|c|c|c|c|c|c|}
\hline & \multirow{2}{*}{\multicolumn{2}{|c|}{ Etapa 1}} & \multicolumn{4}{|c|}{ Etapa 2} & \multirow{2}{*}{\multicolumn{2}{|c|}{ Etapa 3}} & \multirow{2}{*}{\multicolumn{2}{|c|}{ Total }} \\
\hline & & & \multicolumn{2}{|c|}{ Subetapa 2 A } & \multicolumn{2}{|c|}{ Subetapa 2 B } & & & & \\
\hline & $\mathbf{N}$ & $\%$ & $\mathbf{N}$ & $\%$ & $\mathbf{N}$ & $\%$ & $\mathbf{N}$ & $\%$ & $\mathbf{N}$ & $\%$ \\
\hline DT & 3 & $17,65 \%$ & 5 & $29,41 \%$ & 6 & $35,29 \%$ & 3 & $17,65 \%$ & 17 & $100 \%$ \\
\hline TDC & 4 & $23,53 \%$ & 8 & $47,06 \%$ & 2 & $11,76 \%$ & 3 & $17,65 \%$ & 17 & $100 \%$ \\
\hline Total & 7 & $20,59 \%$ & 13 & $38,24 \%$ & 8 & $23,53 \%$ & 6 & $17,65 \%$ & 34 & $100 \%$ \\
\hline
\end{tabular}

Fonte: próprio autor.

intuitivo ao pensamento operatório, caracterizando a construção de forma mais elaborada da operação. Essa característica aparece de maneira evidente nos resultados dos grupos de 6-7 anos, em que algumas crianças com DT $(11,11 \%)$ já se encontram nas etapas finais do desenvolvimento (S2B e E3), enquanto nenhuma das crianças do grupo TDC foi classificada nessas etapas, mantendo-se somente nas etapas iniciais (E1 e S2A).

Esses resultados vão ao encontro do que a literatura específica apresenta, de que o TDC pode estar associado a problemas cognitivos (Skinner \& Piek, 2001, Okuda, 2015) e que, também, possuem baixa capacidade de memória (Wilson, Green, Caeyenberghs, \& Steenbergen, 2016). Vale salientar que crianças com TDC tendem a se afastar da prática de atividades motoras e do convívio social com outras crianças, por perceberem suas próprias limitações, o que pode ser um fator agravante das dificuldades do processo de desenvolvimento desses indivíduos. Desse modo, apesar de a hipótese do estudo não ser confirmada, destaca-se que mais estudos devem ser realizados para investigar a construção dessa noção, principalmente em crianças com TDC.

Em relação aos grupos etários analisados no presente estudo, os resultados se aproximaram das pesquisas de Piaget (2012), no qual 15\% das crianças de seis anos atingiram o pensamento operatório, enquanto $85 \%$ das crianças de 7-8 anos alcançaram este nível do pensamento. Nos resultados aqui encontrados, considerando os dois grupos (TDC e DT), somente $11,11 \%$ de crianças de 6-7 anos classificaram-se na terceira etapa, enquanto $55,00 \%$ de crianças de 8-9 anos atingiram esse nível do desenvolvimento cognitivo. Assim, corroborando os estudos de Piaget, a maior parte das crianças do grupo 6-7 anos apresentaram-se nas etapas inferiores em relação as crianças mais velhas.

Pôde-se observar que um número maior de crianças com TDC do grupo 6-7 anos foi categorizada na etapa E1 (Tabela 3), quando comparado ao grupo DT, o que indica que as crianças com DT alcançam mais cedo as etapas do desenvolvimento cognitivo em relação ao grupo TDC. Contudo, a faixa etária de 8-9 anos não é um marco desenvolvimental de mudança do esquema de conhecimento, pois se encontrou um pequeno percentual $(12,50 \%)$ de crianças com DT dessa faixa de idade classificadas na E1. O fato das crianças não alcançarem a etapa de desenvolvimento na idade esperada é um aspecto variável e está ligado não apenas aos componentes da maturação, mas, também, aos mecanismos internos e externos que influenciam cada indivíduo, às suas interações sociais e às experiências adquiridas nas ações com os objetos (Piaget \& Inhelder, 1986; Piaget, 2012). Nessa mesma perspectiva, Manoel (2000) explica que os caminhos que o desenvolvimento percorre, levando o sistema de um estado a outro, são variados e resultantes da interação entre as restrições do ambiente, do indivíduo e da tarefa.

Ainda, observou-se que, apesar de grande parte das crianças com TDC não alcançar o pensamento operatório dentro da idade esperada, ou seja, aos 6-7 anos de idade, notou-se que entre os 8-9 anos, parte delas atingiu esse nível (30\% no grupo TDC de 8-9 anos). Notou-se, ainda, que um maior número de crianças com

Tabela 3. Número de crianças por grupos em cada etapa.

\begin{tabular}{|c|c|c|c|c|c|c|c|c|c|c|}
\hline & \multirow{2}{*}{\multicolumn{2}{|c|}{ Etapa 1}} & \multicolumn{4}{|c|}{ Etapa 2} & \multirow{2}{*}{\multicolumn{2}{|c|}{ Etapa 3}} & \multirow{2}{*}{\multicolumn{2}{|c|}{ Total }} \\
\hline & & & \multicolumn{2}{|c|}{ Subetapa 2A } & \multicolumn{2}{|c|}{ Subetapa 2B } & & & & \\
\hline & $\mathbf{N}$ & $\%$ & $\mathbf{N}$ & $\%$ & $\mathbf{N}$ & $\%$ & $\mathbf{N}$ & $\%$ & $\mathbf{N}$ & $\%$ \\
\hline DT 6-7 & 2 & $22,22 \%$ & 3 & $33,33 \%$ & 3 & $33,33 \%$ & 1 & $11,11 \%$ & 9 & $100 \%$ \\
\hline DT 8-9 & 1 & $12,50 \%$ & 2 & $25,00 \%$ & 3 & $37,50 \%$ & 2 & $25,00 \%$ & 8 & $100 \%$ \\
\hline TDC 6-7 & 4 & $57,14 \%$ & 3 & $42,86 \%$ & 0 & 0,00 & 0 & 0,00 & 7 & $100 \%$ \\
\hline TDC 8-9 & 0 & 0,00 & 5 & $50,00 \%$ & 2 & $20,00 \%$ & 3 & $30,00 \%$ & 10 & $100 \%$ \\
\hline Total & 7 & $20,59 \%$ & 13 & $38,24 \%$ & 8 & $23,53 \%$ & 6 & $17,65 \%$ & 34 & $100 \%$ \\
\hline
\end{tabular}

Fonte: próprio autor. 
TDC do grupo 8-9 anos foi categorizada na E3, quando comparado ao grupo DT do mesmo grupo etário (Tabela 3), porém, sem se caracterizar como uma diferença estatisticamente significante entre os grupos.

Esses resultados divergem dos achados de um estudo semelhante de Campos, Goldberg, Capellini e Padula (2007), realizado com seis crianças entre 8 e 12 anos, dignosticadas com Transtorno de Déficit de Atenção e Hiperatividade (TDAH), em que foram aplicadas provas operatórias de conservação de quantidades contínuas e descontínuas. As autoras verificaram que as crianças com TDAH se encontravam em um período de desenvolvimento lógico do pensamento anterior ao esperado para a idade. Para explicar os resultados, as autoras citam que a agitação, impulsividade e desconcentração das crianças com TDAH, dificultam a interpretação de problemas, refletindo no comprometimento da aprendizagem.

Entretanto, vale lembrar que as crianças com TDC formam um grupo heterogêneo e que cada uma delas pode apresentar uma variedade diferente de problemas (Okuda, 2015; Yu, Sit, \& Burnett, 2018). Ainda, embora não se tenha clareza sobre a causa do problema relativo ao TDC (Dantas \& Manoel, 2009), estudos apontam que as crianças com transtorno estão mais sujeitas a dificuldades acadêmicas (Michel, Roethlisberger, Neuenscwander, \& Roebers, 2011) e que, geralmente, crianças que sofrem com fracassos constantes acabam não experimentando novas tarefas e, possivelmente, podem abandonar sua vida acadêmica (Santos et al., 2015).

Nesse aspecto, conforme estudo de Medina, Rosa e Marques (2006), no qual investigou-se o desenvolvimento da organização temporal de crianças com dificuldades de aprendizagem entre 8 e 10 anos de idade, pôde-se observar que as crianças com dificuldades de aprendizagem escolar também apresentaram dificuldades nas tarefas motoras com demanda temporal. As autoras verificaram que apenas as crianças de 8 anos apresentaram resultados equivalentes aos níveis esperados para a faixa etária, demonstrando déficit nas diferentes tarefas utilizadas na avaliação. Tais resultados indicaram que, na medida que a idade aumenta, parece haver também um aumento relativo do déficit nos aspectos componentes da organização temporal. Dada essa diferença entre o estudo atual e o estudo de Medina et al. (2006), considera-se necessária a realização de outros estudos que busquem compreender a aprendizagem de aspectos escolares das crianças com dificuldades, principalmente estudos de características longitudinais.

Por outro lado, apesar da carência de estudos que busquem compreender esses aspectos, entende-se que há necessidade de iniciar algum tipo de intervenção o mais cedo possível com essa população, no qual haja o envolvimento da família e, principalmente, do corpo docente, isso porque é no contexto escolar que a criança desenvolve grande parte de suas capacidades motoras e cognitivas (Santos et al., 2015; Pellegrini \& Hiraga, 2008). Nessa perspectiva, estudos de intervenção vêm mostrando influências positivas sobre o desenvolvimento motor destas crianças (Yu et al., 2018, Ferreira, Barros, Bruzi, Santos, \& Freudenhein, 2015). Esses resultados sugerem que profissionais da Educação conheçam o TDC e estejam mais atentos ao desenvolvimento dessas noções, pois este é um processo de reconstrução das estruturas anteriores em um novo plano, com o objetivo de uma reestruturação durante toda a vida do indivíduo (Piaget, 2012). Sendo assim, o conhecimento sobre esse processo se torna imprescíndivel para o planejamento de tarefas que visem as necessidades diferenciadas dos alunos.

As análises e a interpretações das informações deste estudo nos permitiram chegar a três conclusões. Primeira, quanto à noção temporal, os resultados apontaram que as crianças participantes utilizaram a mesma lógica de construção do pensamento quando foram submetidas a um teste observando os materiais (CE) e quando realizaram os movimentos com o próprio corpo, como sujeitos ativos do teste (CA). Segunda, tanto as crianças com TDC quanto as crianças com DT, atingiram os níveis operatórios do pensamento, contudo, em diferentes idades. Terceira, apesar da literatura apontar que, por volta dos 7-8 anos de idade as crianças apresentariam o pensamento operatório, foi possível observar que nem mesmo as crianças com DT atingiram essa etapa.

Como limitações, vale lembrar que esse estudo apresentou uma pequena quantidade de participantes em cada grupo (máximo de 10 crianças por grupo). Isso ocorreu devido a dificuldade de identificação e caracterização de uma amostra maior de crianças com TDC e, todavia isso limitasse as possíveis inferências, todos os critérios propostos na literatura para a identificação desta populaçao foram seguidos. Dessa forma, sugere-se a necessidade de mais estudos com uma quantidade maior de crianças por grupo, a fim de se obter resultados mais robustos em relação ao desenvolvimento cognitivo, e particularmente, como ocorre o desenvolvimento da noção temporal desta população.

Sugere-se, também, que as intervenções no âmbito educacional não se restrinjam às práticas motoras per si, mas avancem para intervenções com atividades de características motoras e cognitivas, com o intuito de auxiliar as crianças a criarem diferentes estratégias de solução dos problemas e, portanto, esquemas de conhecimento mais robustos, minimizando, assim, problemas secundários na vida adulta.

\section{REFERÊNCIAS}

Associação Psiquiátrica Americana. (2014). Manual diagnóstico e estatístico de transtornos mentais (5a. ed.). Porto Alegre: Artmed.

Campos, L. G. A.; Goldberg, T. B. L.; Capellini, A. S.; Padula, N. 
A. M. R. (2007). Caracterização do desempenho de crianças com transtorno de défict de atenção e hiperatividade (TDAH) em provas operatórias: estudos de casos. Revista Psicopedagogia, 24, 218-28.

Cleaton, M. A. M.; Lorgelly, P. K.; Kirby, A. (2019). Developmental coordination disorder: the impact on the family. Quality of Life Research, 28(4): 925-934. DOI:10.1007/s11136-0182075-1

Dantas, L. E. B. P. T.; Manoel, E. J. (2009). Crianças com dificuldades motoras: questões para conceituação do transtorno do desenvolvimento da coordenação. Movimento, 15(3), 293-313.

Estil, L. B.; Ingvaldsen, R. P.; Whiting, H. T. A. (2002). Spatial and temporal constraints on performance in children with movement co-ordination problems. Experimental brain research, 147, 153-161.

Ferreira, L. F.; Barros, J. L. C.; Bruzi, A. T.; Santos, J. O. L.; Freudenhein, A. M. (2015). Transtorno do desenvolvimento da coordenação: discutindo sobre intervenção e seus efeitos. Amazônida, 1, 89-108.

Gimenez, R.; Manoel, E. J.; Oliveira, D. L.; Dantas, L.; Marques, I. (2012). Integrating fundamental movement skills in late childhood. Perceptual and Motor Skills, 114(2), 563-583.

Henderson, S.; Sugden, D. A.; Barnett, A. (2007). Movement Assessment Battery for Children (2. ed.). San Antonio: Harcourt Assessment.

Manoel, E. J. (1994). Desenvolvimento motor: implicações para a educação física escolar I. Revista Paulista de Educação Física, 8(1), 129-147.

Manoel, E. J. (2000). Desenvolvimento motor: padrões em mudança, complexidade crescente. Revista Paulista de Educação Física, 3, 35-54.

Medina, J.; Rosa, G. K. B.; Marques, I. (2006). Desenvolvimento da organização temporal de crianças com dificuldades aprendizagem. Revista da Educação Física/UEM, 17(1), 107-116.

Michel, E.; Roethlisberger, M.; Neuenscwander, R.; Roebers, C. M. (2011). Development of cognitive skills in children with motor coordination impairments at 12-month follow-up. Child Neuropsychology, 17(2), 151-172.

Okuda, P. M. M. (2015). Intervenção e identificação precoce do Transtorno do Desenvolvimento da coordenação em escolares no inicio da alfabetização (Dissertação de mestrado). Universidade Estadual Paulista Julio de Mesquita Filho, Marília, SP, Brasil.

Omer, S.; Jijon, A. M.; Leonard, H. C. (2018). Internalising symptoms in developmental coordination disorder: a systematic review and meta-analysis. Journal of Child Psychology and Psychiatry, 60 (6), 606-621. doi: 10.1111/ jcpp.13001

Pellegrini, A. M.; Hiraga, C. Y. (2008). A intervenção no contexto do comportamento motor: integrando teoria e prática. In Corrêa, U. C. (Ed.), Comportamento motor: a intervenção profissional em perspectiva (116-129). São Paulo: EFP/ EEFEUSP.

Piaget, J. (1958). Psicologia da inteligência (E. de Alencar, trad.). Rio de Janeiro: Fundo de Cultura.

Piaget, J. (1978). Seis estudos de psicologia (24a ed., M. A. M. D’Amorim \& P. S. L. Silva, trad.) Rio de Janeiro: Forense Universitária.

Piaget, J. (2012). A noção de tempo na criança (3a ed., R. Fiúza, trad.) Rio de Janeiro: Civilização Brasileira.

Piaget, J.; Inhelder, B. (1986). A Psicologia da criança (9a ed., O. M. Cajado, trad.) São Paulo: DIFEL S.A.

Poulsen, A. A.; Ziviani J. M.; Johnson H.; Cuskelly M. (2008). Loneliness and life satisfaction of boys with developmental coordination disorder: The impact of leisure participation and perceived freedom in leisure. Human Movement Science, 27(2), 325-43.

Roebers, C. M.; Kauer, M. (2009). Motor and cognitive control in a normative sample of 7-year-olds. Developmental Science, 12, 175-181.

Rosa Neto, F. (2002). Manual de avaliação motora. Porto Alegre: Artmed.

Santos, J. O. L.; Ferreira, L. F.; Souza, M. L.; Branco Junior, A. C.; Oliveira, P. B.; Bruzi, A. T. (2015). Transtorno do desenvolvimento da coordenação: um desafio oculto no cotidiano escolar manauara. Amazônida, 2, 1-15.

Skinner, R. A.; Piek, J.P. (2001). Psychosocial implications of poor motor coordination in children and adolescents. Human Movement Science, 20, 73-94.

Tani, G.; Basso, L.; Corrêa, U.C. (2012). O ensino do esporte para crianças e jovens: considerações sobre uma fase do processo de desenvolvimento motor esquecida. Revista brasileira de Educaçao Física e Esporte, 26(2), 339-50.

Thomas, J. R.; Nelson, J. K.; Silverman, S. J. (2012). Métodos de pesquisa em Atividade Física (6a ed.). Porto Alegre: Artmed.

Wilson, P.; Green, D.; Caeyenberghs, K.; Steenbergen, B. (2016). Integrating New Technologies into the Treatment of CP and DCD. Current developmental disorders reports, 3(2), 138-151.

Yu J. J.; Burnett. A. F.; Sit, C. H. (2018). Motor Skill Interventions in Children with Developmental Coordination Disorder: A Systematic Review and Meta-Analysis. Archives of physical medicine and rehabilitation, 99(10), 2076-2099. doi: 10.1016/j.apmr.2017.12.009

Yu J.; Sit, C. H.; Capio C. M.; Burnett A.; Ha A. S.; Huang W. Y. (2016). Fundamental movement skills proficiency in children with developmental coordination disorder: Does physical self-concept matter? Disability and Rehabilitation, 38(1), 45-51. 